\title{
Happy Hypoxia in COVID-19 Patients at Kinshasa University Hospital (Democratic Republic of the Congo): Frequency and Vital Outcome
}

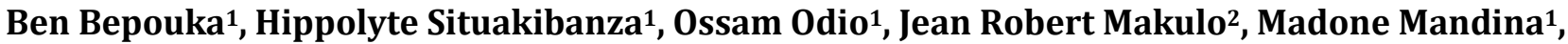
Murielle Longokolo', Nadine Mayasi' ${ }^{1}$, Kazadi Mutombo' ${ }^{1}$, Tresor Pata ${ }^{1}$, Godelive Nsangana ${ }^{1}$, Felly Tshikangu', Donatien Mangala1, Dupont Maheshe', Christine Namasale ${ }^{1}$, Serge Nkarnkwin ${ }^{1}$, Jonathan Muamba', Gorby Ndaie' ${ }^{1}$, Rodrigue Ngwizani ${ }^{3}$, Hervé Mole ${ }^{3}$, Gabriel Makeya ${ }^{3}$, Tharcisse Mabiala3, Patrick Mukuna3, Roger Kabango3, Patricia Kabuni3, Yves Yanga ${ }^{3}$, Aliocha Nkodila ${ }^{4}$, Hervé Keke ${ }^{5}$, Nice Musangu ${ }^{5}$, Papy Tshimanga ${ }^{5}$, Yamin Kokusa1, Bertin Nsitwayizatadi6, Eric Mukenge7, Guyguy Kamwiziku7, Gabriel Mbunsu7, Jean Claude Makangara ${ }^{7}$, Marcel Mbula ${ }^{1}$, Jean Marie Kayembe ${ }^{8}$
\end{abstract}

\footnotetext{
${ }^{1}$ Unit of Infectious Diseases, Kinshasa University Hospital, Kinshasa, Democratic Republic of the Congo

${ }^{2}$ Unit of Nephrology, Kinshasa University Hospital, Kinshasa, Democratic Republic of the Congo

${ }^{3}$ Unit of Reanimation, Kinshasa University Hospital, Kinshasa, Democratic Republic of the Congo

${ }^{4}$ Unit of Vaccinology, World Health Organization, Kinshasa, Democratic Republic of the Congo

${ }^{5}$ Department of Epidemiology, Ministry of Health, Kinshasa, Democratic Republic of the Congo

${ }^{6}$ Unit of Physiotherapy, Kinshasa Hospital University, Kinshasa, Democratic Republic of the Congo

${ }^{7}$ Unit of Microbiology, Kinshasa Hospital University, Kinshasa, Democratic Republic of the Congo

${ }^{8}$ Unit of Pneumology, Kinshasa University Hospital, Kinshasa, Democratic Republic of the Congo

Email: benbepouka@gmail.com
}

How to cite this paper: Bepouka, B., Situakibanza, H., Odio, O., Makulo, J.R., Mandina, M., Longokolo, M., Mayasi, N., Mutombo, K., Pata, T., Nsangana, G., Tshikangu, F., Mangala, D., Maheshe, D., Namasale, C., Nkarnkwin, S., Muamba, J., Ndaie, G., Ngwizani, R., Mole, H., Makeya, G., Mabiala, T., Mukuna, P., Kabango, R, . Kabuni, P., Yanga, Y., Nkodila, A., Keke, H., Musangu, N., Tshimanga, P., Kokusa, Y., Nsitwayizatadi, B., Mukenge, E., Kamwiziku, G., Mbunsu, G., Makangara, J.C., Mbula, M. and Kayembe, J.M. (2021) Happy Hypoxia in COVID-19 Patients at Kinshasa University Hospital (Democratic Republic of the Congo): Frequency and Vital Outcome. Journal of Biosciences and Medicines, 9, 12-20. https://doi.org/10.4236/jbm.2021.92002

\begin{abstract}
Background: Happy hypoxia is a new feature found in COVID-19 patients. It consists of the presence of severe hypoxemia but normal breathing rate. Failure to identify this hypoxia may have negative consequences on the survival of the patient. The objective of the present study was to measure the frequency of patients with happy hypoxia and to evaluate their survival at the Kinshasa University Hospital (KUH). Methods: This was a historical cohort of 141 hospitalized patients with COVID-19 at KUH from March 23 to June 15, 2020. Happy hypoxia was defined as oxygen saturation below $90 \%$ without dyspnea. Socio-demographic data, co-morbidities, follow up time of hospitalization and outcomes were studied. Survival was assessed using the Kaplan Meier curve. Results: Out of 141 hospitalized patients with COVID-19, 79 (56\%) patients were at the severe or critical stage and 9 (6.4\%) had a happy hypoxia on admission. Patients who had happy hypoxia on admission were
\end{abstract}


Received: January 8, 2021

Accepted: February 6, 2021

Published: February 9, 2021

Copyright ( 2021 by author(s) and Scientific Research Publishing Inc. This work is licensed under the Creative Commons Attribution International License (CC BY 4.0).

http://creativecommons.org/licenses/by/4.0/ generally older than 60 years of age $(55.6 \%)(\mathrm{p}=0.023)$. Comparison of survival curves, based on the presence or absence of happy hypoxia, shows a statistically significant difference $(\mathrm{p}=0.001)$. The presence of happy hypoxia reduces survival. Conclusion: The frequency of happy hypoxia among COVID-19 patients was low. Survival was reduced in patients with happy hypoxia. Prehospital pulse oximetry could serve as an early warning signal for the detection of happy hypoxemia in COVID-19 patients.

\section{Keywords}

COVID-19, Happy Hypoxia, Frequency, Outcome, DR Congo

\section{Introduction}

The world is facing a severe pandemic called Coronavirus 2019 Disease (COVID-19) caused by the new Severe Acute Respiratory Syndrome Coronavirus-2 (SARS-COV-2), which broke out in China in December 2019. COVID-19 can be particularly severe and fatal in certain groups such as the elderly or people with co-morbidities. In symptomatic patients, the clinical presentation is mild in about $80 \%$ of cases, moderate to severe in $15 \%$ of cases and critical in $5 \%$ of cases. Patients with severe disease may develop acute respiratory distress syndrome (ARDS), shock, thromboembolic manifestations, multiple organ failure and are at risk of death [1]. Patients with SARS-COV-2 pneumonia may decompensate due to hypoxemic respiratory failure. Autopsy data show diffuse alveolar injury, occasional inflammation, hyaline membranes and accumulation of alveolar fluid, consistent with acute respiratory distress syndrome (ARDS) [2].

One aspect of COVID-19 that puzzles clinicians who must manage pneumonia is that one of the complications of the disease is the presentation of patients with very low blood saturation but no sensation of dyspnea. SARS-COV-2 pneumonia can present clinically in a very heterogeneous fashion. For the same degree of hypoxemia, some patients have a faster breathing rate than others. COVID-19 patients with a very low degree of blood oxygenation (deep hypoxemia) may present very different symptoms. Some may have normal breathing. This phenomenon is called "happy hypoxia" [3]. In the Wuhan cohort of SARS-COV-2 infected patients, only $19 \%$ complained of shortness of breath; $62 \%$ of patients with severe illness and $46 \%$ of those who were intubated, ventilated or died did not have shortness of breath [4]. In cases of happy hypoxia, a persistent normal respiratory rate was inconsistent with the severity of the hypoxia. Roca et al. described the discrepancy between pre-hospital initial respiratory rate (IRR) and initial oxygen saturation $\left(\mathrm{SpO}_{2}\right)$ (i.e., before oxygen supplementation) in COVID-19 patients. They found that some patients had a normal respiratory rate with very low saturation. Measurement of saturation on admission identified non-clinically evident acute respiratory failure [5]. In addition, awake patients who are very ill with COVID-19 therefore require in some re- 
spects as high a level of care as those on mechanical ventilation. Maneuvers that can safely improve oxygenation without requiring additional resources are therefore of immense value during an exacerbation of COVID-19 [6]. COVID-19 patients can rapidly and dangerously desaturate when disconnected from their oxygen source, especially if apparently not in dyspnea. Patients with high oxygen requirements who are managed with alternatives to mechanical ventilation require vigilant monitoring and frequent and careful reassessment [7].

To our knowledge, the socio-demographic and clinical profile and outcome of severe patients with happy hypoxia in Africa subjects are not known. The objective of the study was to measure the frequency of happy hypoxia on admission, to describe the co-morbidities as well as the outcome of these patients who have been hospitalized at the Kinshasa University Hospital.

\section{Materials \& Methods}

This was a historical cohort. It covered the period from March 23 to June 15, 2020. This study was conducted at the Kinshasa University Hospital, a large regional hospital in Kinshasa, DRC. Kinshasa University Hospital is the tertiary level hospital in the city of Kinshasa. This study included all hospitalized patients with COVID-19 confirmed by RT-PCR.

\subsection{Data Collection}

The variables of interest in this study were socio-demographic characteristics (age, sex, education level), co-morbidities (hypertension, diabetes mellitus, asthma, cardiovascular diseases), pregnancy, follow up time of hospitalization and outcome.

\subsection{Operational Definition}

According to some previous study about COVID-19, we define Happy Hypoxia has been defined by an oxygen saturation lower than $90 \%$ on room air without dyspnea with a normal initial respiratory rate at the admission of the patient [7] [8].

\subsection{Laboratory Procedures}

Confirmation of diagnosis

Pharyngeal swabs were taken from each patient for the detection of SARS-COV-2 viral nucleic acids by the RT-PCR assay described above [9].

\subsection{Statistical Analyses}

The analysis was performed with SPSS version 21 software. The descriptive analyses performed were mean, standard deviation for quantitative data with Gaussian distribution and median (IQE = interquartile standard deviation) for data with non-Gaussian distribution. The Pearson Chi-square or Fisher's exact test, as appropriate, was performed to compare proportions. The Kaplan Meier curve 
was used to assess survival. A value of $\mathrm{p}<0.05$ was considered the threshold of significance.

\subsection{Ethical Considerations}

The study was approved by the University of Kinshasa School of Public Health's Ethics Committee (ESP/CE/179/2020). The principle of anonymity and confidentiality was maintained in the study. No conflict of interest was declared in the conduct of this study.

\section{Results}

\subsection{General Characteristics}

Out of 141 hospitalized patients with COVID-19, 79 (56\%) patients were at the severe or critical stage and 9 (6.4\%) had a happy hypoxia on admission (Figure 1). Six patients with happy hypoxia on admission were male $(p=0.963)$. Five patients $(55.6 \%)$ older than 60 years of age $(\mathrm{p}=0.023)$ and seven married patients $(77.8 \%)(\mathrm{p}=0.275)$ had happy hypoxia. No patients with happy hypoxia had comorbidity (Table 1 ).

\subsection{Outcome of Patients with Happy Hypoxia}

Of the 9 patients with happy hypoxia, 4 were deceased and 5 survived. All 4 died within 4 days of hospitalization. Comparison of the survival curves, depending on the presence or absence of happy hypoxia shows a statistically significant difference $(p=0.001)$. The presence of happy hypoxia reduces survival (Figure 2 ).

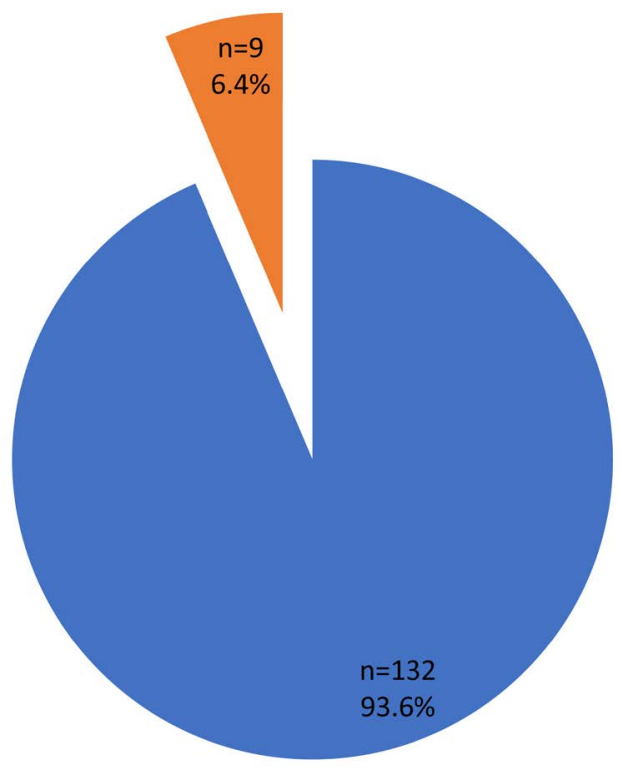

No happy hypoxia $\quad$ Happy hypoxia

Figure 1. Frequency of happy hypoxia at the COVID-19 treatment center of the Kinshasa University Hospital, from March 23rd to June 15th, 2020. 
Table 1. General characteristics of COVID-19 patients at the COVID-19 treatment center of the Kinshasa University Hospital, from March 23rd to June 15th, 2020.

\begin{tabular}{|c|c|c|c|c|}
\hline Variables & $\begin{array}{l}\text { No happy hypoxia } \\
n=132\end{array}$ & $\begin{array}{l}\text { Happy hypoxia } \\
\quad \mathrm{n}=9\end{array}$ & $\begin{array}{l}\text { Over all } \\
\mathrm{n}=141\end{array}$ & $\mathrm{p}$ \\
\hline Sex & & & & 0.963 \\
\hline Male & $89(67.4)$ & $6(66.7)$ & $95(67.4)$ & \\
\hline Female & $43(32.6)$ & $3(33.3)$ & $46(32.6)$ & \\
\hline Age & & & & 0.023 \\
\hline$<40$ ans & $36(27.3)$ & $2(22.2)$ & $38(27.0)$ & \\
\hline $40-59$ ans & $56(42.4)$ & $2(22.2)$ & $58(41.1)$ & \\
\hline$\geq 60$ ans & $40(30.3)$ & $5(55.6)$ & 45 (31.9) & \\
\hline Civil status & & & & 0.275 \\
\hline Married & $82(62.1)$ & $7(77.8)$ & $89(63.1)$ & \\
\hline Single & $39(29.5)$ & $2(22.2)$ & $41(29.1)$ & \\
\hline Widower & $11(8.3)$ & $0(0.0)$ & $11(7.8)$ & \\
\hline \multicolumn{5}{|l|}{ Comorbidities } \\
\hline Hypertension & $33(25.0)$ & $0(0.0)$ & $33(23.4)$ & 0.026 \\
\hline Diabetes mellitus & $24(18.2)$ & $0(0.0)$ & $24(17.0)$ & 0.062 \\
\hline Asthma & $4(3.0)$ & $0(0.0)$ & $4(2.8)$ & 0.596 \\
\hline Cardiovascular diseases & $6(4.5)$ & $0(0.0)$ & $6(4.3)$ & 0.215 \\
\hline Pregnancy & $5(3.8)$ & $0(0.0)$ & $5(3.5)$ & 0.552 \\
\hline
\end{tabular}

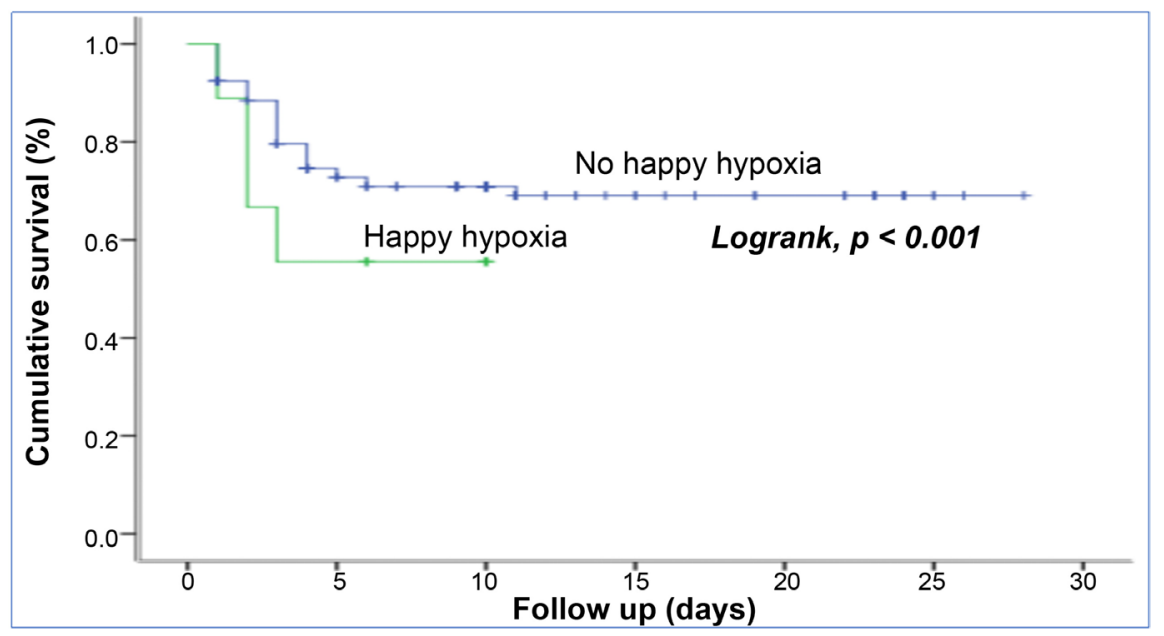

Figure 2. Survival based on happy hypoxia at the COVID-19 treatment center of the Kinshasa University Hospital, from March 23rd to June 15th, 2020.

\section{Discussion}

The frequency of happy hypoxia in this present study was $6.24 \%$. Brouqui et al. [10] found $28.1 \%$ (27/96). The low frequency compared to Brouqui's study would be due among other things to the fact that we considered as threshold a 
saturation lower than $90 \%$ which corresponds to a $\mathrm{PO}_{2}$ of $60 \mathrm{mmHg}$, whereas Brouqui et al. considered $80 \mathrm{mmHg}$ as threshold because the gasometry was performed in all patients.

Happy hypoxia has some explanations in the literature. In situations of happy hypoxia, we found that some patients are tachycardic and have respiratory alkalosis. These signs suppose that part of the sensory information reaches the brain stem to induce a partial compensatory reflex respiratory response and lowers the level of $\mathrm{CO}_{2}$, which diffuses more rapidly in the alveoli than oxygen. The related homeostatic information emanating from the body is part of our interception system, which detects the physiological state of the body, creates consciousness and leads to conscious feelings or symptoms [11]. While in half of ARDS cases the lung loses some of its elastic properties, it remains distensible in the initial stage of COVID-19 pneumonia. This is because the lung retains its ability to vary its volume normally in response to changes in intra-pulmonary pressure. Experts consider this to be "compliance" preserved. This means that the lung retains its elasticity and remains aerated with an amount of intrapulmonary air close to normal while in ARDS outside of COVID-19 the lung becomes stiff. The severe hypoxemia observed in patients with COVID-19 is accompanied by near-normal compliance, especially in the near stage. SARS-COV-2 could also interfere with mitochondrial $\mathrm{O}_{2}$-sensing and causes mitochondrial-induced injury, which impairs carotid body function resulting in impaired respiratory drive and reduced dyspnea [12] [13].

This maintenance of ventilation is associated with the disruption of vascular perfusion due to the prothrombotic phase of COVID-19. Autopsy results for COVID-19 showed the presence of microthrombi in the pulmonary microvasculature [14] [15] [16]. This suggests that happy or silent hypoxemia may be due to conserved ventilation and disrupted perfusion due to capillary obstruction. The anatomical distribution on this peripheral vascular bed undermines the predominantly distal and uneven distribution of radiological infiltrates [17].

This process occurs through projections from the brain stem to the cortex that allows the brain to process homeostatic signals. When hypoxia occurs, the brain receives the internal hypoxic signal, it gives the sensation of "hunger for air" and a need to breathe but in severe COVID-19 patients this sensation is curiously absent. In the presence of hypoxia, respiratory responses occur due to the presence of sensory nerves in the chemoreceptive zones. Changes in the internal environment are recognized by these nerves, they transmit information to the brain stem and these nerves stimulate an increase in the ventilatory drive. Respiratory pathologies provoke auto-nomic reflexes, such as coughing, secretions and bronchospasm. Several clinical conditions can trigger dyspnea. In cardiopulmonary diseases, dyspnea is caused by the entry of multiple homeostatic afferents. The interceptive processing of these signals creates a feeling of breathlessness and a desire to breathe. This primitive brain stem reflex is essential for survival because it can respond to many conditions including hypoxia, acidosis, airway collapse, hypercapnia, irritants and pulmonary vascular congestion [18]. 
Happy hypoxia characterized by the dissociation between the absence of dyspnea and the profound hypoxemia does not have clear pathophysiological mechanisms. Among the reasons evoked, there are also lesions of the vagus and glossopharyngeal nerves after neck cancer, a congenital neuropathy. In the context of COVID-19, the possibility that SARS-COV-2 has neuroinvasive action is controversial. On the one hand, there is the presence of neurological signs such as convulsions, delirium, altered mental status and anosmia. The possible damage to neurons could be due to the direct action of SARS-COV-2 on nerve fibres and the intense cytokine storm after the inflammatory phase. On the other hand, SARS-COV-2 could enter the brain through pathways connected to synapses and is found in the cerebral fluid of the spinal column [19]. On the other hand, the results of brain magnetic resonance imaging (MRI) studies and pathology reports in fatal cases of COVID-19 are inconsistent and do not provide a pathophysiological correlation to explain the absence of dyspnea. The common brain pathology findings in fatal COVID-19 cases are multiple areas of ischemic stroke and hemorrhagic microbleeding with only small areas of inflammation; however, it should be noted that at least $40 \%$ of the brain imaging studies were normal and there were no signals of brain stem abnormalities on MRI scans [20].

Regardless of the uncertain underlying pathology, reduced perception of dyspnea is a disorder of blood gas interception. It can mask the severity of the condition and ultimately delay the time at which the patient can seek urgent medical attention. Patients admitted with COVID-19 may suffer sudden death after voluntary "cuts" in oxygen supplementation. No patient with happy hypoxia had comorbidity. The small number of patients with happy hypoxia in this study does not allow us to confirm this assertion. The data in the literature to date do not provide a correlation between happy hypoxia and comorbidities. Happy hypoxia reduced survival. This result is similar to the study by Brouqui $e t$ al. who found that happy hypoxia was associated with poor outcomes (33.3\% were transferred to the intensive care unit and $25.9 \%$ died) [10]. These patients who do not have warning signs of poor outcome could be falsely considered to be clinically well, and caregivers may be distracted leading to a negative outcome if monitoring is not rigorous.

\section{Limitations}

The study was retrospective with the possibility of missing data. We did not measure hypoxia by gasometry by looking for the partial pressure of oxygen but by pulse oximetry. The small sample size of patients with happy hypoxia limit did us to better establish the characteristics of this new entity.

\section{Conclusion}

Happy hypoxia was present in our cohort. The frequency of happy hypoxia among COVID-19 patients was low. Elderly people were predominant. Survival was reduced in patients with happy hypoxia. Prehospital pulse oximetry could 
serve as an early warning signal for the detection of happy hypoxemia in patients with COVID-19. Continued research is important to establish the link between happy hypoxia and advanced age.

\section{Acknowledgements}

The authors acknowledge ALIMA NGO for support in the management of COVID-19 patients, especially Drs Armand, Christian MASUDI and Junior MUWALAWALA. I acknowledge also ANDEMIA project for epistat and scientific writing capacity building.

\section{Conflicts of Interest}

All authors declare no conflicts of interest.

\section{References}

[1] Wu, Z. and McGoogan, J.M. (2020) Characteristics of and Important Lessons from the Coronavirus Disease 2019 (COVID-19) Outbreak in China: Summary of a Report of 72314 Cases from the Chinese Center for Disease Control and Prevention. JAMA, 323, 1239-1242.

[2] Archer, S.L., Sharp, W.W. and Weir, E.K. (2020) Differentiating COVID-19 Pneumonia from Acute Respiratory Distress Syndrome (ARDS) and High-Altitude Pulmonary Edema (HAPE): Therapeutic Implications. Circulation, 142, 101-104. https://doi.org/10.1161/CIRCULATIONAHA.120.047915

[3] Couzin-Frankel, J. (2020) The Mystery of the Pandemic's "Happy Hypoxia". Science, 368, 455-456.

[4] Guan, W.-J., Ni, Z.-Y., Hu, Y., et al. (2020) Clinical Characteristics of Coronavirus Disease 2019 in China. The New England Journal of Medicine, 382, 1708-1720. https://doi.org/10.1056/NEJMoa2002032

[5] Roca, O., Messika, J., Caralt, B., et al. (2016) Predicting Success of High-Flow Nasal Cannula in Pneumonia Patients with Hypoxemic Respiratory Failure: The Utility of the ROX Index. Journal of Critical Care, 35, 200-205. https://doi.org/10.1016/j.jcrc.2016.05.022

[6] World Health Organization (2020) Clinical Management of Severe Acute Respiratory Infection (SARI) When COVID-19 Disease Is Suspected. https://www.who.int/docs/default-source/coronaviruse/clinical-management-of-no vel-cov.pdf

[7] Caputo, N.D., Strayer, R.J. and Levitan, R. (2020) Early Self-Proning in Awake, Non-Intubated Patients in the Emergency Department: A Single ED's Experience during the COVID-19 Pandemic. Academic Emergency Medicine, 27, 375-378. https://doi.org/10.1111/acem.13994

[8] Widysanto, A., Wahyuni, T., Simanjuntak, L., et al. (2020) Happy Hypoxia in Critical COVID-19 Patient: A Case Report in Tangerang, Indonesia. Physiological Reports, 8, e14619. https://doi.org/10.14814/phy2.14619

[9] World Health Organization (2020) Coronavirus Disease (COVID-19) Technical Guidance: Laboratory Testing for 2019-nCoV in Humans.

https://www.who.int/emergencies/diseases/novel-coronavirus-2019/technical-guida nce/laboratory-guidance

[10] Brouqui, P., Amrane, S., Million, M., et al. (2020) Asymptomatic Hypoxia in 
COVID-19 Is Associated with Poor Outcome. International Journal of Infectious Diseases, 102, 233-238. https://doi.org/10.1016/j.ijid.2020.10.067

[11] Robinson, D. and Gebhart, F. (2008) Inside Information: The Unique Features of Visceral Sensation. Molecular Interventions, 8, 242-253. https://doi.org/10.1124/mi.8.5.9

[12] Gattinoni, L., Coppola, S., Cressoni, M., et al. (2020) COVID-19 Does Not Lead to a “Typical” Acute Respiratory Distress Syndrome. American Journal of Respiratory and Critical Care Medicine, 201, 1299-1300.

[13] Negri, E.M., et al. (2020) Heparin Therapy Improving Hypoxia in COVID-19 Patients-A Case Series. https://www.medrxiv.org/content/10.1101/2020.04.15.20067017v3

[14] Tian, S., Hu, N. and Niu, L. (2020) Pulmonary Pathology of Early-Phase 2019 Novel Coronavirus. Pneumonia in Two Patients with Lung Cancer. Journal of Thoracic Oncology, 15, 700-704. https://doi.org/10.20944/preprints202002.0220.v2

[15] Yao, X.H., Li, T.Y., He, Z.C., et al. (2020) A Pathological Report of Three COVID-19 Cases by Minimally Invasive Autopsies. Chinese Journal of Pathology, 49, 411-417.

[16] Dolhnikoff, M., Duarte-Neto, A.N., de Almeida Monteiro, R.A., et al. (2020) Pathological Evidence of Pulmonary Thrombotic Phenomena in Severe COVID-19. Journal of Thrombosis and Haemostasis, 18, 1517-1519. https://doi.org/10.1111/jth.14844

[17] Ye, Z., Zhang, Y., Wang, Y., et al. (2019) Chest CT Manifestations of New Coronavirus Disease.

[18] Burki, N. and Lee, L. (2010) Mechanisms of Dyspnea. Chest, 138, 1196-1201. https://doi.org/10.1378/chest.10-0534

[19] Duarte, A.G. and Kauffmann, L.N. (2020) Is "Happy Hypoxia” in COVID-19 a Disorder of Autonomic Interoception? A Hypothesis. Clinical Autonomic Research, 30, 331-333.

[20] Coolen, T., Lolli, V. and Sadeghi, N. (2020) Early Postmortem Brain MRI Findings in COVID-19 Non-Survivors. Neurology, 95, e2016-e2027.

https://doi.org/10.1101/2020.05.04.20090316 